\title{
Inexpensive and easy method for 6 fragment Golden Gate Assembly of a modular S/MARs mammalian expression vector and its variants
}

\author{
Authors: IONUT ADRIAN PASCU ${ }^{*}$ \\ 1 Faculty of Biology, University of Bucharest, Bucharest, Romania \\ *Author to whom correspondence should be addressed: E-mail: ionut.cepleanu@gmail.com
}

\begin{abstract}
Background

A basic requirement for synthetic biology is the availability of efficient DNA assembly methods. Numerous methods have been previously reported to accomplish this task. One such method has been reported, which allows parallel assembly of multiple DNA fragments in a one-tube reaction, called Golden Gate Assembly. This study aims to further simplify that method and make it more suitable for small labs and students.
\end{abstract}

\section{Methods}

Prior to amplification of the parental plasmids used in building the modules were domesticated using a variation of SDM (Site Directed Mutagenesis) called SPRIP. After careful design and amplification of the desired modules, using a high fidelity polymerase, amplified PCR fragments that enter the one-step-one-pot reaction were stored in Zymo DNA/RNA Shield at -20 degrees C and thawed whenever needed to be used as fragments or modules in the assembly. The fragments were designed to posses unique overhangs using NEB Golden Gate assembly tool and Snapgene , amplification of modules was performed using a Q5 high fidelity polymerase from preexisting plasmids or gene fragments, clean-up of the PCR products (fragments) was performed in one tube per assembly using Zymo DNA Clean and Concentrator-5, assembled using BsaI and T4 ligase, DpnI digestion performed for eliminating the background plasmids that remain after the PCR reaction and the resulting assembled product was transformed into competent E.coli cells. Transformants were screened using diagnostic digest and reporter gene expression in both bacteria and HEK293 cells was evaluated using fluorescent light.

\section{Results}

Herein presented is a simple and inexpensive alternate protocol to build modular plasmids using the Golden Gate Assembly method. Four S/MARs mammalian expression vectors were designed 
and constructed using 6 modules previously amplified by PCR and stored in the appropriate buffer to eliminate exo- and endonuclease activity and to protect the DNA from freeze thaw cycles. The existing modules were interchangeable and new modules were easily amplified and stored for use when needed. The mammalian expression vectors constructed showed the desired restriction pattern and GFP expression in bacteria and in mammalian cells

\section{Conclusions}

Provided the design steps are respected and the fragments are stored and labeled appropriately , multiple plasmid variants and combinations of the pre-designed modules can be assembled in one day, easier and using less resources than the established protocols, with good efficiency. The simplicity of the design and the affordability of the method could make modular cloning of plasmid constructs more accessible to small labs and students.

\section{Key words: Golden Gate, Cloning, S/MARS, mammalian expression vector}

\section{Background}

The principle of Golden Gate Cloning[1] is based on the ability of type IIS enzymes to cleave outside of their recognition site, allowing two DNA fragments flanked by compatible restriction sites to be digested and ligated seamlessly. Since the ligated product of interest does not contain the original type IIS recognition site, it will not be subject to redigestion in a restrictionligation reaction.

All products that reconstitute the original site will be re-digested, allowing their components to be made available for further ligation, leading to formation of an increasing amount of the desired product with increasing time and cycles of incubation. Since the sequence of the overhangs at the ends of the digested fragments can be chosen to be any 4-nucleotide sequence, multiple compatible DNA fragments can be assembled in a defined linear order in a single restriction-ligation step.

Based on this method multiple standards to construct multi-gene circuits have been developed. Mo-Clo, or the modular cloning standard, is one of these standards [2]. Mo-Clo consists of standard parts, or basic modules, of eukaryotic Promoters, 5'UTR's, Signal Peptides, CDS's, 3'UTR's and Terminator regions, each module containing a standardized 4 nucleotide overhang. Mo-clo or similar standards like Golden Braid 2.0 [3] use 3 levels of successive assemblies. 
Level 0, or basic modules (Promoters, 5'UTR's, Signal Peptides, CDC's, 3'UTR's and Terminator regions) are assembled into Level 1 transcriptional units using a Type IIs restriction enzyme. The level 1 transcriptional units can be, in turn, ligated into level 2 multigene constructs using a different type IIs restriction enzyme.

However, although standardized and agreed by the community, these standards may prove too complex for assembling just a few/several fragments or for students that are just getting started in molecular biology or that have a desire for more customization.

In turn, here described is a simple inexpensive protocol for standardizing and assembling several fragments with good fidelity, in just one day with minimal hands-on time.

For this purpose a modular S/MARs mammalian expression vector (called pNoname) has been designed. A S/MARs vector is a type of non-integrating episomal vector that is used for mammalian expression[4][5]. It contains a region called a Scaffold/Matrix Attachment Region which is an element designed to permit the persistence of the vector as an extrachromosomal element within a host cell [6]. It also provides the vector with the ability to remain episomal and be mitotically stable during cell division [5]. Furthermore, these types of vectors can replicate during cell division in certain types of cells. [7].

The modular vector designed in this paper contains 6 fragments or modules that can be easily interchangeable.

In order to facilitate the Golden Gate Assembly, fragments to enter the assembly, previously amplified by PCR can be stored in Zymo DNA/RNA Shield at a ratio of 1:3 (PCR : Zymo Shield) thus eliminating the need to store the fragments in plasmids and facilitating the screening of the desired mutants.

Zymo DNA/RNA shield protects DNA from freeze thaw cycles, stabilizes nucleic acids and deactivates nucleases. [8]. DNA overhangs of several bases designed in primers can further protect the PCR products from exonucleases thus leaving the type IIs restriction sytes intact.

After designing the primers to possess specific 4 base pair overhangs and accommodating the DNA fragments that will enter the assembly reaction to lack any other specific type IIs restriction sites (depending on the type IIs restriction enzyme used) [9] the fragments can be cleaned, assembled and the resulting product transformed into competent E.coli cells (home-made or purchased) to generate the desired mutants. 
Multiple plasmids can be constructed at the same time providing that certain rules are followed and provided that the correct fragments are mixed for each reaction.

Fragment assemblies (called F or modules) and transformations can be performed in the same day. The core fragment which contains the bacterial origin of replication and the antibiotic gene can be amplified by PCR. Multiple types of core fragments could be used, with interchangeable antibiotic resistance genes. Of the amplified PCR products stored in ZS (Zymo Shield), $20 \mu \mathrm{l}$ can be added to the assembly reaction (of each fragment type). Before assembly, the PCR mix (mixed fragments) is cleaned and concentrated and $20 \mu \mathrm{l}$ of eluate is used in the GG assembly reaction. Multiple reactions can be made at the same time. After the assembly, DpnI digestion is performed to remove the background plasmid that was used as a PCR template and then $2-5 \mu \mathrm{l}$ is transformed into competent E.coli.

\section{Methods}

\section{pNoname design and construction}

In order to provide a backbone for future experiments a modular S/MARs vector called pNoname was designed. All in silico designs were made using the Snapgene software [10]. The vector consists of 6 modules, designed to be easily interchangeable in a short amount of time and with minimum financial effort.

1) One module consists of the required bacterial origin of replication for replication and maintenance in bacteria and the antibiotic resistance gene for selection.

2) One module consists of the promoter and enhancer region.

3) One module consists of the reporter gene (cGFP) with a Kozack consensus sequence.[11]

4) One module consists of a shortened Nuclear Matrix Attachment Region, MARS5 [7] directly downstream of the gene.

5) One module consists of the Terminator and the poly adenylation sequence.

6) One module consists of the SV40 (Simian Virus 40) DTS (DNA nuclear targeting sequence), a $72 \mathrm{bp}$ repeat of the early enhancer, that has been shown to mediate nuclear transport of the inserted DNA. [12]

The primers for amplifying the fragments or modules were designed using the New England Biolabs Golden Gate Assembly Tool [13] in order to generate specific 4 base pairs overhangs and were further modified to accommodate other requirements (GC content, primer TM 
etc). The standardized 4 base pairs overhangs are highlighted in Figure 1. After digestion with BsaI and ligation with T4 ligase the BsaI recognition sequence is eliminated from the final construct thus preventing redigestion. The primers used for the amplification of the modules are shown in Table 1.

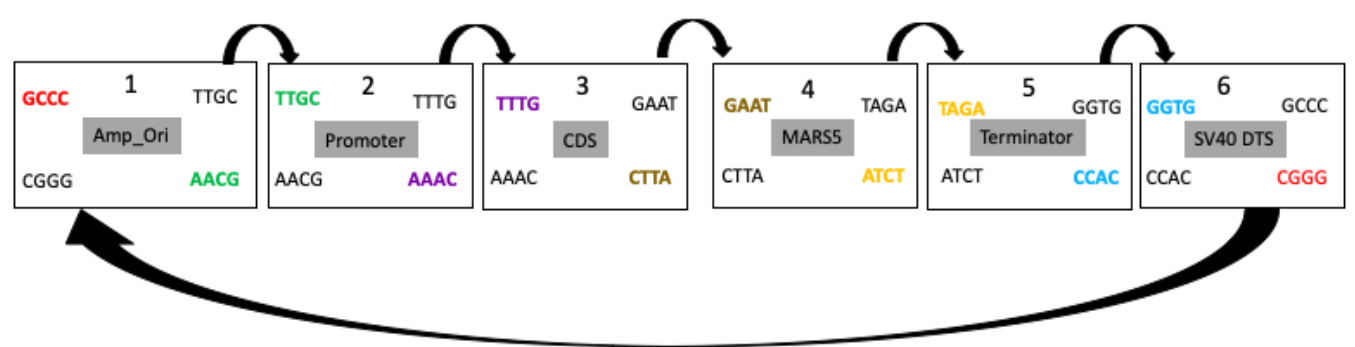

Figure. 1 Overhangs generated for each module with the assemble direction given in arrows.

A schematic representation of pNoname variant 1 (pNoname_CMV_GFP_BGH) is shown in Figure 2.

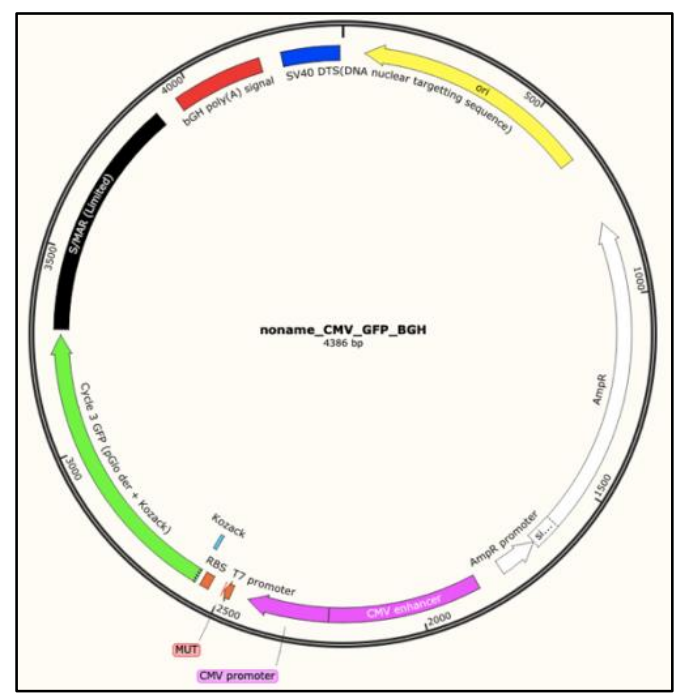

Figure 2. pNoname variant CMV_GFP_BGH after assembly that contains all 6 modules (Promoter region, Coding seqence, MARS5, Terminator and poly adenilation signal, SV4O DTS and the core fragment (bacterial origin of replication and antibiotic resistance gene)

\section{Domestication (removal of internal BsaI sites)}

In order to enter the Golden Gate Assembly, all other BsaI sites except the ones containing the overhangs must be eliminated from the fragments.

Domestication of the plasmids was performed using primers FPCTokillmyBsaI, RPCTokillmyBsaI, FTokillBsaI, RtokillBsaI (provided in [Additional file 3]) with a modified 
version of Site Directed Mutagenesis, called SPRIP (Single Primer Reaction in Parallel) [14]. Two plasmids were domesticated, pUC18 and pcDNA3.1(+) (ThermoFischer Cat.No V79020). The primers were designed to be complementary to each other and to contain a single base pair mutation that will eliminate the BsaI from the ampicilin resistance gene of the pUC18 plasmid and eliminate the BsaI site just downstream of the T7 promoter of pcDNA3.1(+) respectively. Single primer PCR was performed in 2 different tubes for each plasmid using Q5 2X Master Mix(New England Biolabs Cat.No M0492S). After the amplification, the PCR tubes contents were mixed and allow to re-anneal using the following cycle $\left(5 \mathrm{~min}\right.$ at $95^{\circ} \mathrm{C} ; 1 \mathrm{~min}$ at $90^{\circ} \mathrm{C} ; 1 \mathrm{~min}$ at $80^{\circ} \mathrm{C}$, $0.5 \mathrm{~min}$ at $70^{\circ} \mathrm{C}, 0.5 \mathrm{~min}$ at $60^{\circ} \mathrm{C}, 0.5 \mathrm{~min}$ at $50^{\circ} \mathrm{C}, 0.5 \mathrm{~min}$ at $40^{\circ} \mathrm{C}$, hold at $37^{\circ} \mathrm{C}$ ). After the reannealing step, DpnI (New England Biolabs Cat.No R0176S) digestion was performed prior to transformation to eliminate the parental plasmid. Transformed cells (New England Biolabs E.coli Turbo Cat.No) made competent using the protocol provided in [Additional file 1] were placed in the incubator overnight at $37^{\circ}$ and colonies were screened for desired mutants using BsaI-HF®v2 (New England Biolabs Cat.No R3733S).

\section{Construction of modules (fragments) for assembly}

The Amp_Ori region was amplified from pUC18 after it's domestication (removal of any internal BsaI sites). The other modules used in this experiment, CMV promoter and Enhancer, the SV40 promoter, the SV40-DTS, the BGH terminator region and the SV40 terminator region were amplified from pcDNA3.1(+) after domestication.

\begin{tabular}{|c|c|c|c|c|}
\hline Amp+Ori_fwd_pUc18 & $5^{\prime}-3^{\prime}$ & ggctacggtctcagcccctaactcCAGCAAAAGGCCAGGAACC & 43 & GCCC \\
\hline Amp+Ori_rev_pcUc18 & $5^{\prime}-3^{\prime}$ & ggctacggtctctgcaaGTCGACGTCAGGTGGCAC & 35 & gcaa \\
\hline Promoter_CMV_fwd & $5^{\prime}-3^{\prime}$ & ggctacggtctct ttgcTTCGCGATGTACGGG & 32 & $\operatorname{ttgc}$ \\
\hline Promoter_CMV_rev & $5^{\prime}-3^{\prime}$ & ggctacggtctca caaaattatttcAAGTTTAAACGCTAGCCAGCT & 48 & caaa \\
\hline GFPKozack_fwd & $5^{\prime}-3^{\prime}$ & ggctacggtctcaTTTGTTTAACTTTAAGAAGGAGATATAC & 41 & tttg \\
\hline GFPKozack_rev & $5^{\prime}-3^{\prime}$ & ggctacggtctcCATTCATTATTTGTAGAGCTCATC & 36 & attc \\
\hline MARSS_fwd & $5^{\prime}-3^{\prime}$ & ggctacggtctccgaattcgagctcCTGTGCTGTCGCCTAACTATC & 46 & gaat \\
\hline MARS5_rev & $5^{\prime}-3^{\prime}$ & ggctacggtctcatctagactcgagTCATGGGATCCAAGTATCAAG & 46 & tcta \\
\hline TERM_BGH_fwda & $5^{\prime}-3^{\prime}$ & ggctacggtctca TAGAGGGCCCGTTTAAACC & 32 & taga \\
\hline TERM_BGH_rev & $5^{\prime}-3^{\prime}$ & ggctacggtctcg ccacagaatAGCCCCAGCTGGTTCTTTC & 41 & ccac \\
\hline TERM_SV40_fwd & $5^{\prime}-3^{\prime}$ & ggctacggtctcaTAGACTCATGCTGGAGTTCTTCG & 35 & taga \\
\hline TERM_SV40_Rev & $5^{\prime}-3^{\prime}$ & ggctacggtctcgccacTGACCATGATTACGCCAAGC & 37 & ccac \\
\hline PROMO_SV40_FWd & $5^{\prime}-3^{\prime}$ & ggctacggtctcg TTGCAATGTGTGTCAGTTAGGG & 35 & $\operatorname{ttg} c$ \\
\hline PROMO_SV40_ReV & $5^{\prime}-3^{\prime}$ & ggctacggtctca caaa aCCTGTCTCTTGATCAGATC & 37 & caaa \\
\hline DTS40_fwd & $5^{\prime}-3^{\prime}$ & ggctacggtctcgGTGGAATGTGTGTCAGTTAGGG & 35 & gtgg \\
\hline DTS40_rev & $5^{\prime}-3^{\prime}$ & ggctacggtctcaGGGCGGGACTATGGTTGC & 31 & $g g g c$ \\
\hline
\end{tabular}

Table 1. Primers used in building the fragments for GGA (Golden Gate Assembly). In bold and coloured black the $B$ sal restriction site contained in each primer. The 4 base pair overhang providing direction in assembly is shown in italic. Shown in brown is the region that anneals to the template. 
Gfp_Kozack (The reporter gene coding for a green fluorescent protein) and MARS5 were synthetized by Twist Biosciences. Primers were used to further multiply the sequences from pNoname.

All amplifications were made using a high fidelity polymerase (New England Biolabs Q5 2X Master Mix) according to the manufacturers recommendations in a $25 \mu$ l final reaction volume.

\section{Construction of pNoname variants}

After amplification, $20 \mu 1$ of the PCR product that contained the specific modules were stored in Zymo DNA/RNA shield (Zymo Research Cat.No R1100-50) in the freezer at -20 degrees Celsius, at a ratio of 1:3 (DNA : Zymo Shield / $20 \mu 1$ DNA:60 $\mu 1$ Zymo Shield). $5 \mu 1$ of the PCR product was loaded on a gel for confirmation of the reaction. $20 \mu 1$ of each module (PCR product + Zymo Shield) (6 in total) were mixed in a single tube and cleaned using the Zymo Research DNA Clean and Concentrator -5 kit (Cat.No D4013). Each tube contained fragments from a single pNoname variant. A total of 4 pNoname variants were constructed. (pNoname_CMV_GFP_BGH, pNoname_SV40_GFP_SV40, pNoname_CMV_GFP_SV40, pNoname_SV40_GFP_BGH). The assembly reaction was set up as follows: $20 \mu 1$ of mixed purified fragments (6 fragments in total) , $1.5 \mu \mathrm{l}$ (600 U) of T4 ligase (New England Biolabs Cat.No M0202S), 1 l (20 U) of BsaI-HF®v2, 2,5 $\mu 1$ of T4 Ligase Buffer. $5 \mu l$ of purified fragments were kept for gel agarose confirmation. The assembly cycle was: $\left(1 \mathrm{~min}\right.$ at $16^{\circ} \mathrm{C}+1 \mathrm{~min}$ at $\left.37^{\circ} \mathrm{C}\right) \mathrm{X} 30$ cycles followed by a 5 minute soak time at $60^{\circ} \mathrm{C}$. DpnI digestion was performed to eliminate any background plasmids from the PCR reaction using $1 \mu \mathrm{l}$ of $\mathrm{DpnI}\left(37^{\circ} \mathrm{C}\right.$ for 15 minutes followed by 5 minutes at $\left.60^{\circ} \mathrm{C}\right)$ and transformed with $5 \mu \mathrm{l}$ of assembly product using E.coli Neb Turbo made competent by the transformation protocol in Supplemental Materials. Cells were plated on antibiotic selection plates with LB Agar containing $100 \mu \mathrm{g} / \mathrm{ml}$ ampicilin and left in the incubator overnight at $37^{\circ} \mathrm{C}$.

Mutants were confirmed by restriction digest on a $1 \%$ agarose gel and by amplifying the regions using primers provided in Table 1.

The detailed design and assembly protocol is provided in [Additional file 2]. 


\section{Results}

\section{Domestication (removal of internal BsaI sites)}

After the restriction digest BsaI should not be able to linearize the pUC18-BsaI mutant, thus, compared to the wild type variant the mutant should migrate as the supercoiled form in the gel. pcDNA3.1(+) contains two BsaI restriction sites. The mutant form, pcDNA3.1(+)-BsaI, after domestication, should migrate as one linearized fragment of 5428 base pairs on the gel, as opposed to the wild type variant that should generate 2 fragments. The results of the restriction digest are shown in Figure 3 and Figure 4.

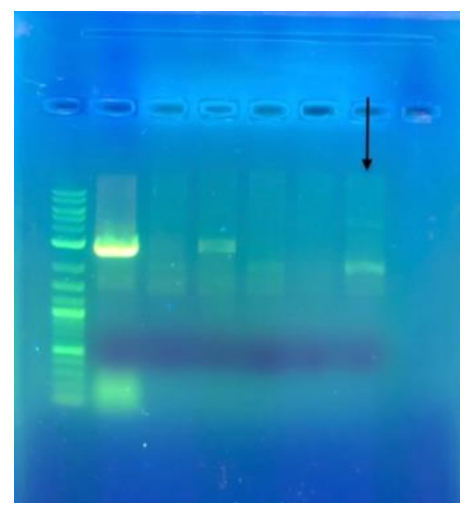

Figure 3. Gel electrophoresis after Bsal restriction digest. Lane 1 Ladder $1 \mathrm{~kb}$ plus New England Biolabs, Lane 2 wild type pUC18 (Control), lanes $3,4,5,6,7$ screened colonies after miniprep digested with Bsal. Lane 7 shows the desired pattern.

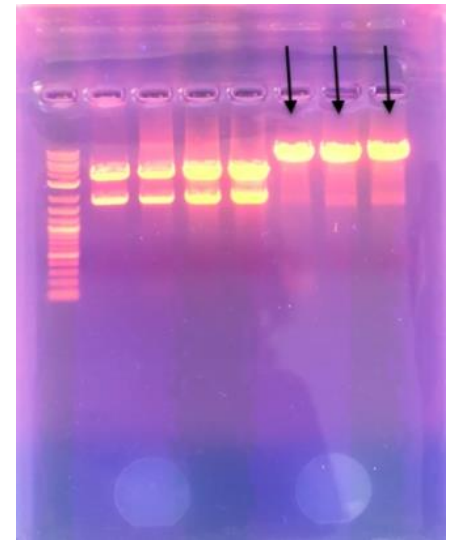

Figure 4. Gel electrophoresis after Bsal restriction digest. Lane 1 Ladder 1 kb plus New England Biolabs, Lane 2 wild type pcDNA3.1(+) (Control), lanes $3,4,5,6,7,8$ screened colonies after miniprep digested with Bsal.

\section{Construction of modules (fragments) for assembly}

The fragments (modules) needed for assembly were amplified using a high fidelity polymerase in a final reaction volume of $25 \mu 1$, according to the suppliers recommendations. $5 \mu 1$ of each reaction was loaded onto a $1 \%$ agarose gel next do a DNA ladder to confirm successful amplification. $20 \mu 1$ of each reaction was stored at a ratio of 1:3 in Zymo DNA/RNA Shield and aftert proper labelling, the tubes were placed in the freezer at $-20^{\circ}$.

\section{Construction of pNoname variants}

Four pNoname variants were constructed in the same day using $20 \mu$ l of each module previously amplified and stored in Zymo Shield. The six modules were loaded in each of the 4 
tubes corresponding to each variant to assemble. A gel electrophoresis pre and post assembly confirmed the successful assembly of the variants (Figure 5). Data not shown for pNoname_CMV_GFP_SV40 and pNoname_CMV_GFP_BGH.

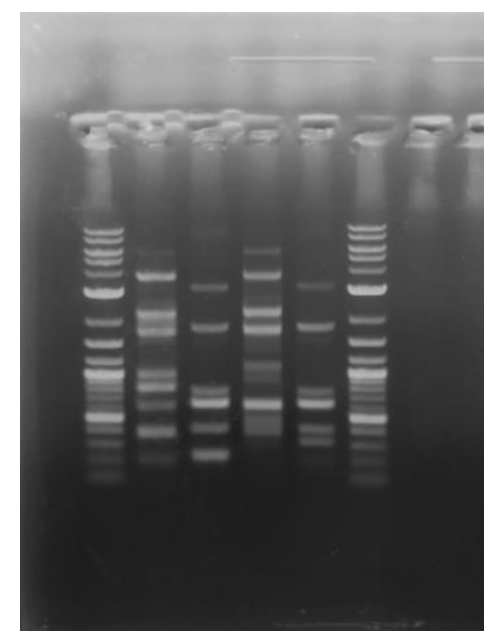

Figure 5. Lane 1 Ladder $1 \mathrm{~kb}$ plus New England Biolabs, Lane 2 pNoname_SV40_GFP_SV40 post assembly, Lane 3 pNoname_SV4O_GFP_SV4O pre assembly, Lane 4 pNoname_SV4O_GFP_BGH post assembly, pNoname_SV4O_GFP_BGH pre assembly

After transformation 2-4 colonies from each of the four plates were harvested for screening. Desired mutants were confirmed by restriction digest according to their individual DNA sequences. Out of 2 mutants screened for pNoname_SV40_GFP_SV40 both showed the restriction pattern consistent with a correct assembly. Out of $\mathbf{2}$ mutants screened for pNoname_SV40_GFP_BGH $\underline{\text { one }}$ showed the desired pattern. For pNoname_CMV_GFP_BGH 3 out of 4 colonies screened showed the desired pattern and 1 out of $\mathbf{2}$ colonies screened showed the desired pattern for CMV_GFP_SV40 respectively (data not shown). Although few colonies were screened, mutants were confirmed in all plates that were processed. (Figure 6 and Figure 7).

The number of colonies obtained after the transformation with the assembled DNA was low, for all 4 plasmid variants, especially considering the fact that the whole outgrowth after the 1 $\mathrm{h}$ incubation step at $37^{\circ} \mathrm{C}$ was concentrated (outgrowth was spinned at 1000G for 6 minutes and the bacterial pellet resuspended in $100 \mu \mathrm{l}$ of LB Broth and plated; The detailed transformation procedure is provided in Additional file 2. 
bioRxiv preprint doi: https://doi.org/10.1101/2021.09.04.458594; this version posted September 11, 2021. The copyright holder for this preprint (which was not certified by peer review) is the author/funder, who has granted bioRxiv a license to display the preprint in perpetuity. It is made available under aCC-BY-NC 4.0 International license.

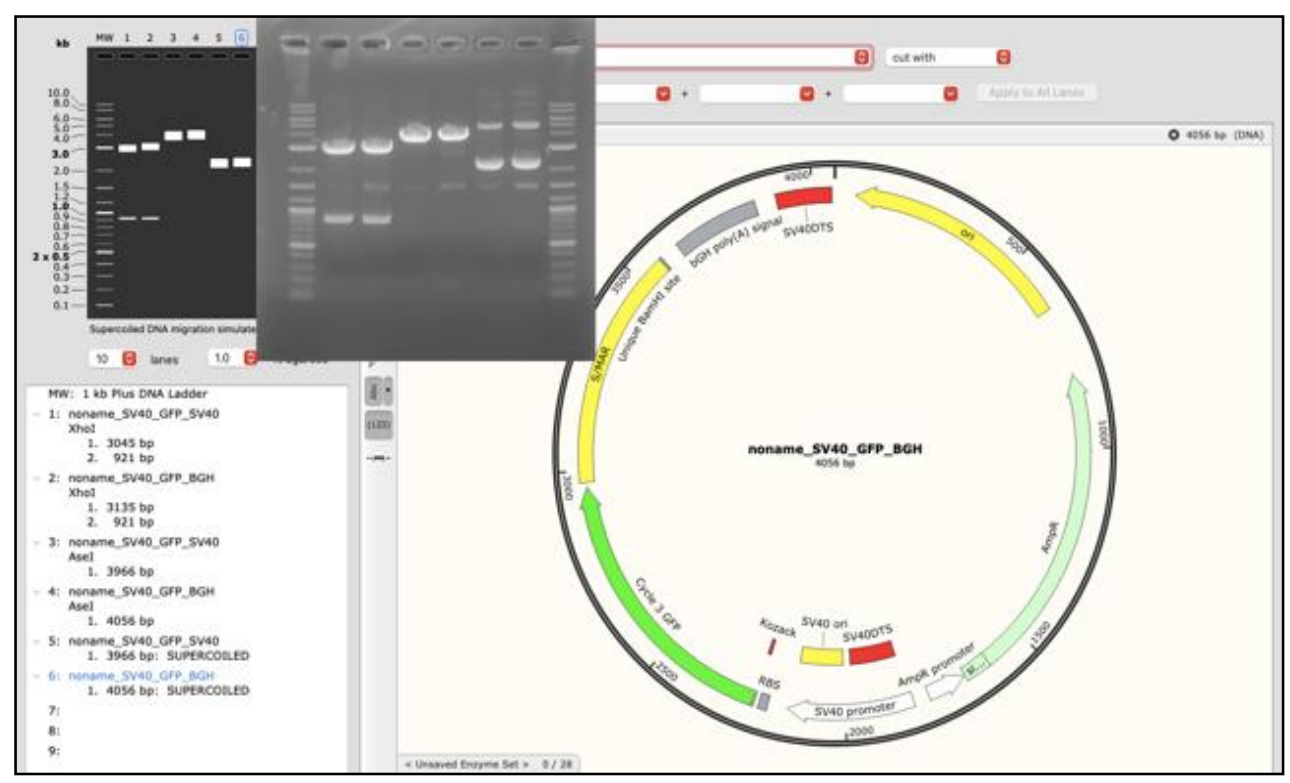

Figure 6. pNoname variants. Lane 1 Ladder $1 \mathrm{~kb}$ plus New England Biolabs, Lane 2 pNoname_SV4O_GFP_SV4O digested with Xhol, Lane 3 pNoname_SV4O_GFP_BGH digested with Xhol, Lane 4 pNoname_SV4O_GFP_SV40 digested with Asel, Lane 5 pNoname_SV4O_GFP_BGH digested with Asel, Lane 6 pNoname_SV4O_GFP_SV4O non-digested, Lane 7 pNoname_SV4O_GFP_BGH non-digested, Lane 8 Ladder $1 \mathrm{~kb}$ plus New England Biolabs

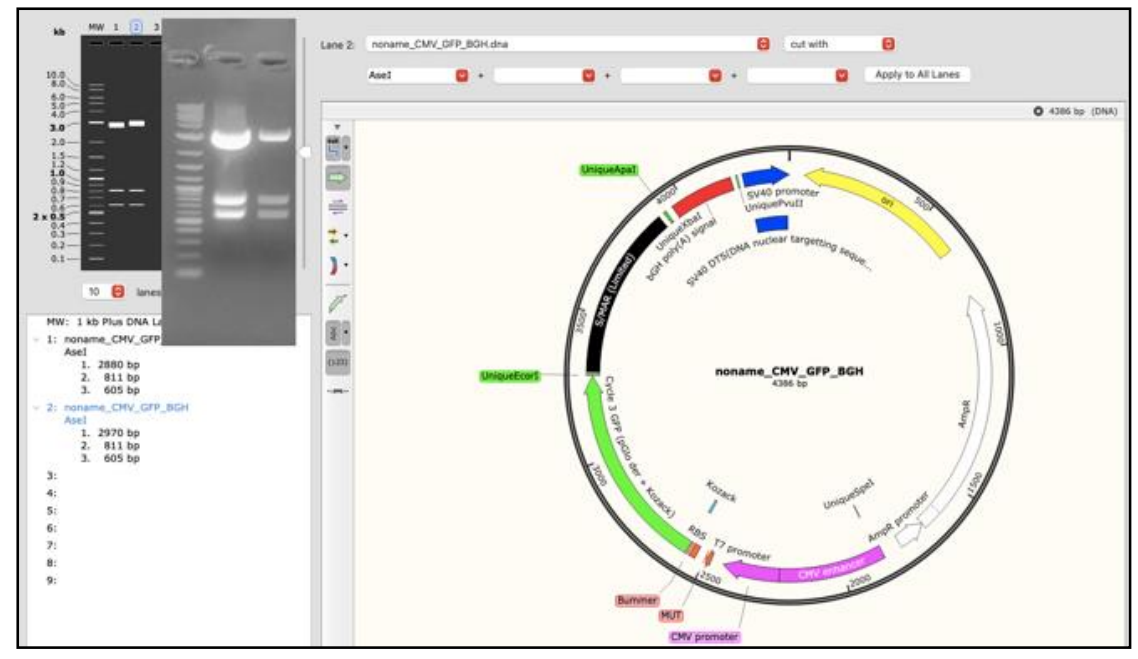

Figure 7. pNoname variants. Lane 1 Ladder $1 \mathrm{~kb}$ plus New England Biolabs, Lane 2 pNoname_CMV_GFP_SV4O digested with Asel, Lane 3 pNoname_CMV_GFP_BGH digested with Asel 
$5 \mu \mathrm{l}$ of transformed pNoname_SV40_GFP_SV40 generated 25 colonies per plate after transformation, $5 \mu$ l of pNoname_SV40_GFP_BGH generated 28 colonies after transformation into home-made competent E.coli (Figure 8).

Similarly, pNoname_CMV_GFP_SV40 and pNoname_CMV_GFP_BGH generated approximately the same number of colonies after transformation with $5 \mu \mathrm{l}$ of assembled product. (Data not shown).

However, this fact (low transformation efficiency) did not present itself as an inconvenience due to the fact that assembly efficiency was high, considering the ratio of screened correct/incorrect assembled mutants.

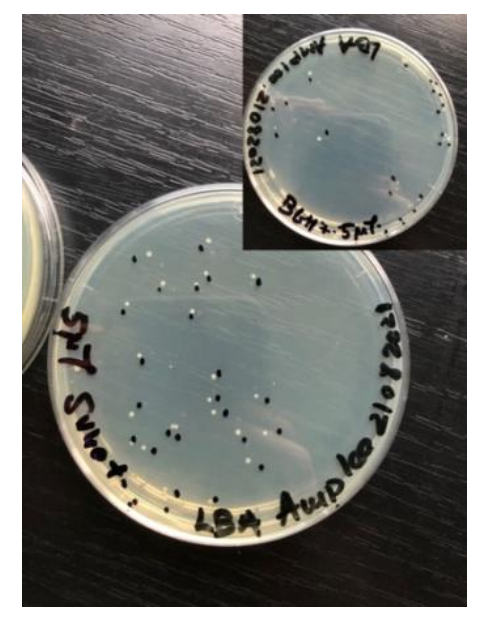

Figure 8. Colonies generated after transforming E.coli Neb Turbo with $5 \mu$ l of assembled pNoname_SV40_GFP_SV40 and $5 \mu$ l of pNoname_SV4O_GFP_BGH. 27 and 25 colonies per plate respectively

\section{pNoname_CMV_GFP_BGH fluorescent expression testing.}

The pNoname variants CMV_GFP_BGH and CMV_GFP_SV40 (the variants that contain the CMV enhancer and promoter regions) were designed to contain a T7 promoter for ease of screening in bacteria prior to transfection in mammalian cells.

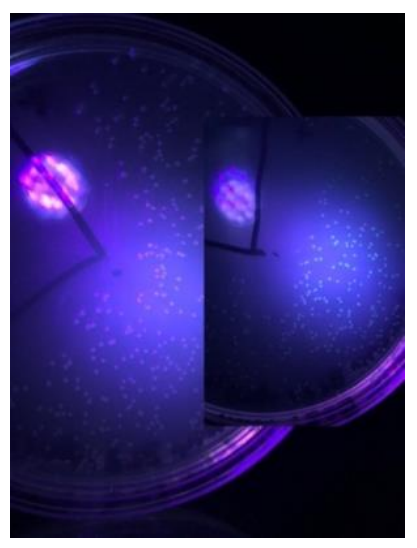

Figure 9. pNoname_CMV_GFP_BGH transfromed in E.coli BL21(DE3). Induced vs. Non-induced with IPTG under UV flashlight 
The T7 promoter is a sequence of DNA 18 base pairs long up to transcription start site at $+1\left(5^{\prime}-\right.$ TAATACGACTCACTATAG - 3') that is recognized by T7 RNA polymerase [15]. It's induction with IPTG is possible when the vector that contains it is expressed in a bacterial strain specifically engineered to code for T7 RNA polymerase, such as E.coli B121(DE3). E.coli B121(DE3) posesses the lacUV5 promoter that expresses the T7 RNA polymerase upon induction with IPTG. Results show that induction of pNoname_CMV_GFP_BGH promotes GFP expression when transformed in BL21(DE3) compared to it's non-IPTG induced counterpart, albeit the expression is not a strong one, no doubt also limited by the lack of a proper bacterial terminator.

When transfected in mammalian HEK293T with PEI the EGFP expression is present although no further experiments beyond screening for mammalian expression using a fluorescent microscope have been made at this point.

The ability of the vector to be maintained as an episome inside HEK293 cells, the transfection efficiency and the intensity of the fluorescence have not been evaluated. The cells have not been passaged and the fluorescence generated by the cGFP gene was not monitored beyond the 120 hour mark.

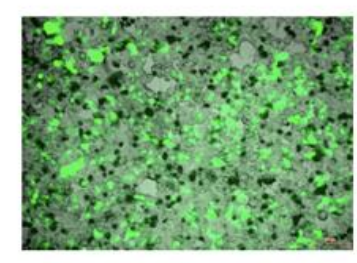

Day 1

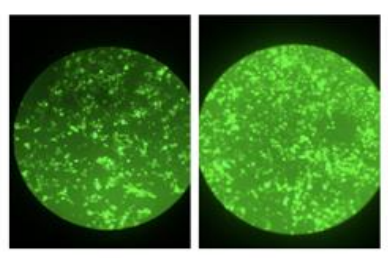

Day 2

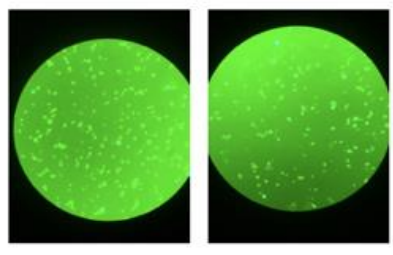

Day 4
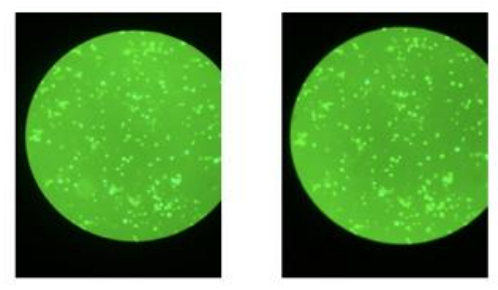

Day 5

Figure 10. HEK293T cells transfected with pNoname_CMV_GFP_BGH using PEI. Expression was monitored using a fluorescence microscope at day 1, day 2, day 4 and day 5 post transfection. 


\section{Discussion}

The need for cheap, easy methods to design and build various expression vectors is still present in molecular and synthetic biology, especially for small laboratories that lack the capacity to implement large scale screening and/or construction of many vectors at a time and complicated gene circuits. By careful design and with cost savings in mind, small laboratories and students can build vectors without the need for multiple restriction enzymes, laborious screening and using just a few selected products.

The protocol presented here could be such an alternative. Counterintuitively, by building the plasmids from the ground up every time instead of cloning a single gene, using stored modules amplified by PCR (including the core element consisting of the bacterial origin of replication and the antibiotic resistance gene), instead of using the concept of vectors and inserts, the workflow could be simplified.

The design could be adapted easily to accommodate more/less modules. Using just two primers designed to possess the right overhangs one or more fragment types could be eliminated from the final construct with ease.

The fact that equimolar amounts of both fragments and ,vector backbone” could be introduced into the assembly reaction and the amount of DNA could be approximated by gel electrophoresis alone, would eliminate the use of a spectrophotometer.

Storing the amplified PCR fragments or modules in an environment that is endo-and exonuclease free, protecting the nucleic acids from freeze thaw cycles, amplifying further modules with a high fidelity polymerase that has proof reading capabilities when needed using just $1 \mu l$ of parental plasmid, cleaning the PCR products just one time per assembly reaction, customizing the modules through careful design and using just one restriction enzyme for molecular cloning could be a reliable and cheap method for multiple modular vector construction in a very short time, reducing hand-on time and costs.

\section{Conclusions}

This method demonstrates that modular expression vectors could be constructed easily, affordably and with minimal hands-on time by even the most under funded and under equipped 
laboratories. By carefully optimizing the design and methods used, multiple modular expression vectors with interchangeable parts can be build by anyone using minimum equipment and reagents.

\section{Abbreviations}

Amp_Ori - ampicilin resitance gene and bacterial origin of replication

BGH - The bovine growth hormone polyadenylation (bgh-PolyA) signal

CDS - coding sequence

cGFP - cycle Green Fluorescence Protein

CMV - cytomegalovirus major immediate early enhancer

SDM - Site directed mutagenesis

S/MARs - scaffold/matrix attachement region

SPRIP - Single Primer Reaction in Parallel

SV40 promoter - early promoter of the Simian Virus 40

SV40 terminator - Simian Virus 40 polyadenilation sequence

$\mathrm{TM}$ - primer melting temperature

3'UTR - 3' untranslated region

\section{Declarations}

\section{Ethical Approval and Consent to participate}

Not applicable

\section{Consent for publication}

Not applicable

\section{Availability of data and materials}

All data generated or analysed during this study are included in this published article [and its supplementary information files]

\section{Competing interests}


The authors declare that they have no competing interests

\section{Funding}

Funding was provided by the author

\section{Authors' contributions}

IAP performed all designs and experiments in this study

\section{Acknowledgements:}

I would like to express my very great appreciation to Sebastian.S Cocioba for providing guidance and the bacterial strains used in this paper, to David Ishee for providing the first version of the bacterial transformation protocol used, to Lector Dr. Ionela Sarbu for providing the primers used and to Dr. Rodica-Aura Badea for the help provided in testing pNoname expression in HEK293 cells. I would also like to express my profound appreciation to prof. Dr. Ileana Stoica whose guidance and support has been invaluable.

\section{REFERENCE}

1. Engler C, Kandzia R, Marillonnet S (2008) A One Pot, One Step, Precision Cloning Method with High Throughput Capability. PLoS ONE 3:e3647. https://doi.org/10.1371/journal.pone.0003647

2. Weber E, Engler C, Gruetzner R, et al (2011) A Modular Cloning System for Standardized Assembly of Multigene Constructs. PLOS ONE 6:e16765. https://doi.org/10.1371/journal.pone.0016765

3. Sarrion-Perdigones A, Falconi EE, Zandalinas SI, et al (2011) GoldenBraid: An Iterative Cloning System for Standardized Assembly of Reusable Genetic Modules. PLOS ONE 6:e21622. https://doi.org/10.1371/journal.pone.0021622

4. Argyros O, Wong S, Niceta M, et al (2008) Persistent episomal transgene expression in liver following delivery of a scaffold/matrix attachment region containing non-viral vector. Gene Ther 13

5. Argyros O, Wong SP, Fedonidis C, et al (2011) Development of S/MAR minicircles for enhanced and persistent transgene expression in the mouse liver. J Mol Med 89:515-529. https://doi.org/10.1007/s00109-0100713-3

6. Hagedorn C, Schnödt-Fuchs M, Boehme P, et al (2017) S/MAR Element Facilitates Episomal Long-Term Persistence of Adeno-Associated Virus Vector Genomes in Proliferating Cells. Hum Gene Ther 28:1169-1179. https://doi.org/10.1089/hum.2017.025 
7. Wang X-Y, Zhang X, Wang T-Y, et al (2019) Shortened nuclear matrix attachment regions are sufficient for replication and maintenance of episomes in mammalian cells. Mol Biol Cell 30:2761-2770. https://doi.org/10.1091/mbc.E19-02-0108

8. Prevent Nucleic Acid Degradation Before Extraction. In: ZYMO Res. https://www.zymoresearch.com/blogs/blog/prevent-nucleic-acid-degradation. Accessed 3 Sep 2021

9. Marillonnet S, Grützner R (2020) Synthetic DNA Assembly Using Golden Gate Cloning and the Hierarchical Modular Cloning Pipeline. Curr Protoc Mol Biol 130:e115. https://doi.org/10.1002/cpmb.115

10. SnapGene | Software for everyday molecular biology. In: SnapGene. https://www.snapgene.com/. Accessed 8 Sep 2021

11. Kozak M (1986) Point mutations define a sequence flanking the AUG initiator codon that modulates translation by eukaryotic ribosomes. Cell 44:283-292. https://doi.org/10.1016/0092-8674(86)90762-2

12. Dean DA, Dean BS, Muller S, Smith LC (1999) Sequence Requirements for Plasmid Nuclear Import. Exp Cell Res 253:713-722. https://doi.org/10.1006/excr.1999.4716

13. NEB Golden Gate. https://goldengate.neb.com/\#!/. Accessed 1 Sep 2021

14. Edelheit O, Hanukoglu A, Hanukoglu I (2009) Simple and efficient site-directed mutagenesis using two singleprimer reactions in parallel to generate mutants for protein structure-function studies. BMC Biotechnol 9:61. https://doi.org/10.1186/1472-6750-9-61

15. Rong M, He B, McAllister WT, Durbin RK (1998) Promoter specificity determinants of T7 RNA polymerase. Proc Natl Acad Sci 95:515-519. https://doi.org/10.1073/pnas.95.2.515 